\title{
Optimal Approach for Enhancement of Large and Small Scale Near-Infrared and Infrared Imaging
}

\author{
Zhengmao Ye, Jiecai Luo, Pradeep Bhattacharya \\ Electrical Engineering Department \\ Southern University \\ Baton Rouge, LA70813, USA
}

\begin{abstract}
In a broad area of industry such as remote sensing and medical diagnosing, imaging enhancement technology takes a leading role, where energy distribution of the light source depends not only on image coordinate but also on wavelength. Both infrared (IR) and near-infrared (NIR) imaging techniques have a variety of applications in these fields. For instance, satellite images are taken via IR or NIR spectrometer and laser Doppler medical scanning is collaborated with NIR spectrometer. Matrix functions of any image correspond to brightness or energy at each image pixel. The actual decision making must rely on detailed investigation of images being obtained. Therefore, image processing should be taken into account so as to enhance the results from real world. Segmentation is an image analysis approach to clarify feature ambiguity and information noise, which divides an image into separate parts that correlate with the objects or areas of the particular object involved. This procedure can be conducted by clustering, which is a process of partitioning a set of pattern vectors into subsets. Being a simple unsupervised learning algorithm, K-means clustering algorithm has the potential to both simplify the computation and accelerate the convergence. In most cases optimization is closely related to clustering, which gives rise to the best way of problem solving. In this article, optimal approach is proposed to be implemented along with image segmentation. This methodology is to enhance both large scale and small scale IR and NIR image processing.
\end{abstract}

\section{INTRODUCTION}

Image processing technology deals with producing and reestablishing an array of pixels for object representation in order to enhance raw images from direct measurements. Natural world is intrinsically 3D, while human perceptions are mostly 2D. The $2 \mathrm{D}$ and $3 \mathrm{D}$ information are normally expressed as brightness values, bounded by certain limits. In image acquisition of remote sensing, each satellite platform is characterized by wavelengths, sensory spatial resolution, coverage areas and temporal converges. The outcomes from spatial remote sensing represent the large scale imaging. On the contrary, most medical pattern recognition systems lead to small scale imaging. The principles of diverse imaging systems also vary a lot. For example, in terms of resolution, imaging systems can be classified into low resolution, medium resolution, high resolution and very high resolution systems; in terms of spectral regions, imaging systems can be classified into X-ray, visible, near infrared and infrared systems, etc. Processing of remote spatial images involves large-scale spatial data management, which is very complex and highly nonlinear. On the other hand, for some fields of clinical therapy, there is a need for an accurate numerical measurement and a pictorial representation to overcome the problem of spot measurements and allow assessment of the whole region, such as skin depth measurement using a scanning laser Doppler technique together with NIR spectroscopy. In both cases, there is a necessity of information exploitation in order to group pixels together into regions of similarity, according to the changing rate of depth (3D) on the image or according to changing rate of intensity (2D) over a region. In general, among basic requirements of image processing, e.g., noise smoothing, clustering, segmentation, contrast stretching, information restoration, etc, image segmentation is an appropriate option to amalgamate similar pixels to form a set of coherent image layers or regions from any individual image together.

The goal of segmentation is to classify an image into parts that have a strong correlation with objects to reflect the real world information. Thus any raw image is decomposed into recognized physical entities for further analysis. Clustering is one of the primary global approaches of segmentation which depends on partition of images into a set of layers or regions. The image segmentation by K-Means clustering readily refers to grouping similar data points into individual clusters. Sometimes a simplified K-means clustering case can be used to reduce computational complexity. There are several optimum statistical classifier algorithms that can be selected, such as threshold based, region based, edge based or surface based. Optimal algorithm needs to be conducted so as to obtain the best clustering result, such as nearest neighbor rule or winner-take-all (WTA) algorithm [1-13]. The content of this work is focused on image enhancement of both large scale and small scale NIR and IR images. 


\section{Principle of SATEllite AND Doppler IMAging}

Infrared detector is a dominant technology for biomedical non-destructive diagnosis and testing as well as for night vision. Detection of infrared spectrum is basically classified into two major categories: photonic based or thermal based.

Satellite imaging system is capable of transmitting both near infrared (NIR) and short-wave infrared (SWIR) as well as visible light. The advantage lies in that infrared channel is selected exclusively at night and is used simultaneously with visible channel during the day, thus information can be captured at anytime daily, regardless of weather condition. Operating principle and structure of the satellite imaging system are shown in Fig. 1, where a set of telescopes, NIR spectrometer, SWIR spectrometer and visible spectrometer can be used together. Large scale spatial IR and NIR images may be obtained from this system, which are usually used for forecasting, monitoring and navigating.

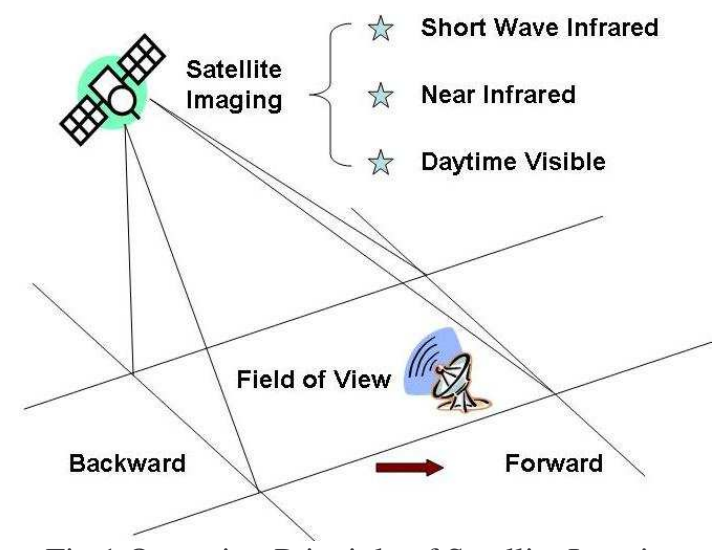

Fig.1 Operating Principle of Satellite Imaging

On the contrary, small scale near infrared image can be captured by laser Doppler scanning system, which is used to exert a laser beam onto skin or tissue surface for imaging and medical diagnosis. Flowing blood causes Doppler shift of the scattered laser light. The shift information is detected and processed using near infrared (NIR) spectrometer. The measurement is non-contact and real-time which quantify differences in blood flow and assess flow patterns over time. The corresponding color images of blood flow are indicated from the computer (Fig. 2). Doppler scanning system can be directly applied on medical diagnosis of burn depth of skin.

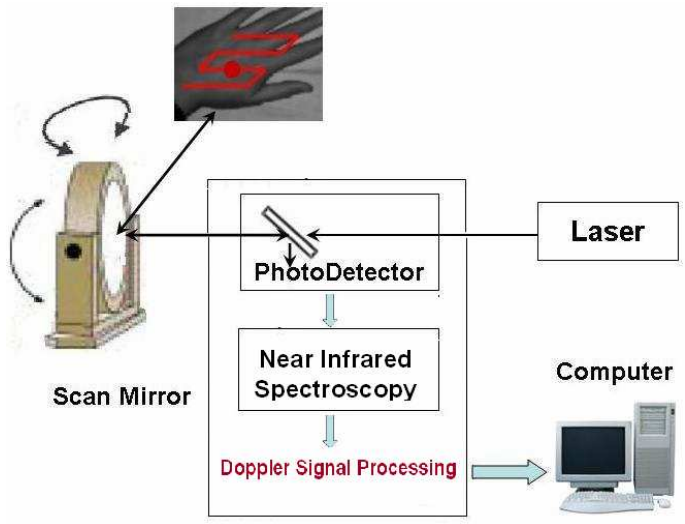

Fig.2 Laser Doppler Scanning and NIR Spectroscopy

\section{UNSUPERVISED IMAGE SEGMENTATION}

Image segmentation acts as a useful resource to uncover object contents using data analysis. The common way is to use either edge-based or region-based algorithm to solve a complicated problem. Due to varied nature, two algorithms may produce different results. Most potential imaging issues are in need of certain segmentation method. The fact is that there is no universal approach that could be proper for all segmentation possibilities. For any segmentation approach, both global segmentation and partial segmentation can be selected. Global approach uses color histogram of image features as a basis of segmentation. Each layer is uniquely associated with particular object from the original image. Partial segmentation does not associate with image objects in a regular way. Image is divided into various parts that are homogeneous with respect to property of brightness, color and texture, etc. In this research, a global approach using color histogram of image features is selected. The information of brightness is recorded as a basic factor for segmentation. For satellite IR/NIR images, segmentation helps to compensate for distortion caused by atmosphere dispersing on actual scenes. For medical 2D and 3D NIR images, segmentation helps to capture abnormal symptoms for accurate diagnosis. Unsupervised clustering technology is thereby proposed for image processing since no explicit teacher is available. Image enhancement has been conducted in spatial domain for both large-scale satellite IR/NIR data and small-scale medical Doppler NIR data.

In order to effectively synthesize all essential information, $\mathrm{K}$-means clustering is presented so as to separate dissimilar details. K-means clustering requires that individual number of clusters be specified for partitioning and distance metric be defined to quantify relative distance of objects for pattern recognition and optimization. In general, elements can be conjoined together to represent an image pixel or partially used to some extent. Then imaging system forms clusters of input patterns. Diversified image features are investigated in terms of unsupervised methods. To reduce the computation complexity, simplified clustering should be implemented in certain cases. For clustering procedures, optimal approach should be conducted to achieve the final decision making on a basis of the specified distance metric being defined. Euclidean distance and Mahalanobis distance are two major types of distance metrics, which are used for clustering. The former method is straightforward and the latter one encloses the information into its covariance matrix. A simple cluster separation can be judged by nearest neighbor rule, while in other cases, optimization method should be considered such as the competitive learning algorithm of winner-take-all rule. The contrasted objects on a uniform environment directly lead to the segmentation problems. Accordingly, contrast stretching is applied prior to the image retrieval procedure. Hence, the significant signatures from images will be emphasized, from which accurate decision making can be made. 


\section{Color Histogram Based K-Means Clustering}

Cluster analysis divides sample data into several groups so that similar data objects belong to the same cluster and different data objects belong to separate clusters. K-means clustering follows an easy way to classify the data sets through $\mathrm{K}$ number of clusters. Initially, all color histograms to be used are normalized. Then an image is partitioned into $\mathrm{n} \times \mathrm{m}$ smaller subsets using Quadtree method with varying squares, which gives rise to satisfied results (Fig. 3).

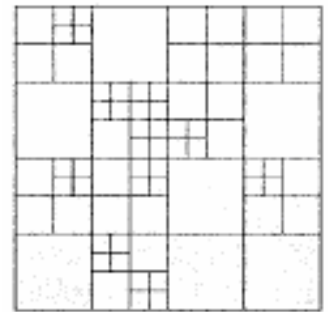

Fig.3 Partitioning via Quadtree

Now define centers of each cluster as $C_{i}$. The procedure classifies the data sets into $\mathrm{K}$ number of clusters. The center of each cluster is the mean value of those data points which belong to the same cluster. Following this procedure, a distance measurement $D(x, y)$ is defined to be similarity measurement. The distances of a data point to these cluster centers are compared such that a data point belongs to its most adjacent cluster via nearest neighbor rule.

$l_{k}\left(x_{k}\right)=\arg \min _{i} D\left(x_{k}, C_{i}\right)=\arg \min _{i}\left\|x_{k}-C_{i}\right\|^{2}$

where $l_{k}$ is the label for the data point $x_{k}$. The K-means algorithm tries to find a set of cluster centers so that the total distortion is minimized. Here, image distortion is defined by the total summation of the distances of data points from its cluster center (2).

$$
\varphi(\mathcal{X}, \mathcal{C})=\sum_{i \in \mathcal{C}} \sum_{i \in \text { i-th cluster }}\left\|x_{j}-C_{i}\right\|^{2}
$$

To minimize the distortion $\phi$, K-means algorithm iterates between labeling and re-centering steps.

For labeling, assume the $p$-th iteration ends up with a set of cluster centers $C_{i}^{(p)}, i=1,2, \ldots K$. We label each data point based on such a set of cluster centers, i.e., $\forall x_{k}$, find its $l_{k}^{(p+1)}\left(x_{k}\right)=\min _{i}\left\|x_{k}-C_{i}^{(p)}\right\|^{2}$

and group data points belong to the same cluster $\Omega_{\mathrm{i}}=\left\{\mathrm{x}_{\mathrm{k}}: \mathrm{l}_{\mathrm{k}}\left(\mathrm{x}_{\mathrm{k}}\right)=\mathrm{C}_{\mathrm{i}}\right\}$

For re-centering, calculate the centers for all the clusters $C_{i}^{(p+1)}=\frac{\sum_{x_{k} \in \Omega_{i}} x_{k}}{\left|\Omega_{i}\right|}$

This algorithm iterates between labeling and re-centering procedures until it converges to the local stationary.

\section{Optimal Processing Study of SAtellite Imaging}

In this section, two cases are to be studied for satellite image clustering and segmentation. A regular K-Means clustering using nearest neighbor rule and color histogram method will be conducted at first. Afterwards, the reduced K-Means clustering case using winner-take-all algorithm will be conducted, followed by contrast stretching.

\section{A. Large-Scale Image Segmentation $(30 m \times 30 m / p i x e l)$}

Fig.4 - Fig.8 show the image clustering and segmentation results of Oak Ridge area. These are still raw results as the segmented areas are within 30 meter image. The idea is to identify and partition each type of objected individually to obtain the refining results. Different features of spatial color histogram, spatial color histogram varying differences and spatial color histogram shape can be reconsidered as a new efficient retrieval feature index. Processing results show that both water regions and tree regions are clearly recognized.

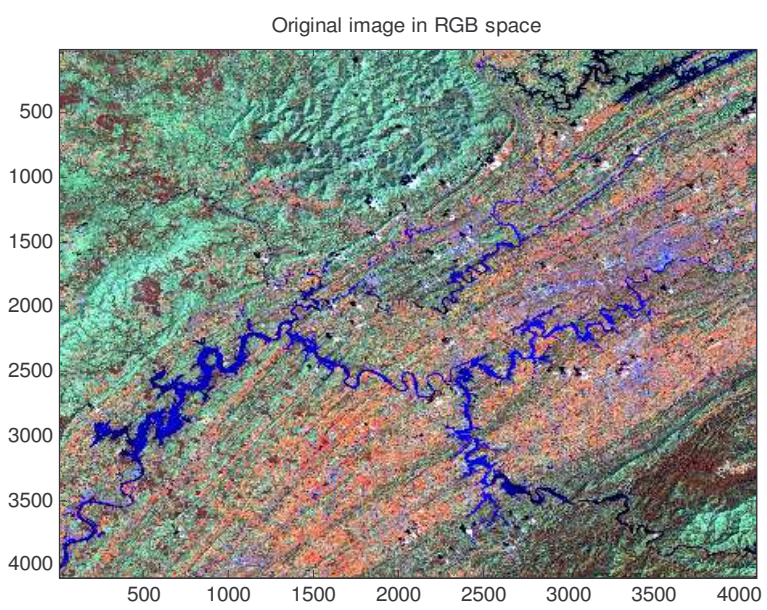

Fig.4 Original Oak Ridge Image $(30 \mathrm{~m} \times 30 \mathrm{~m} /$ pixel $)$

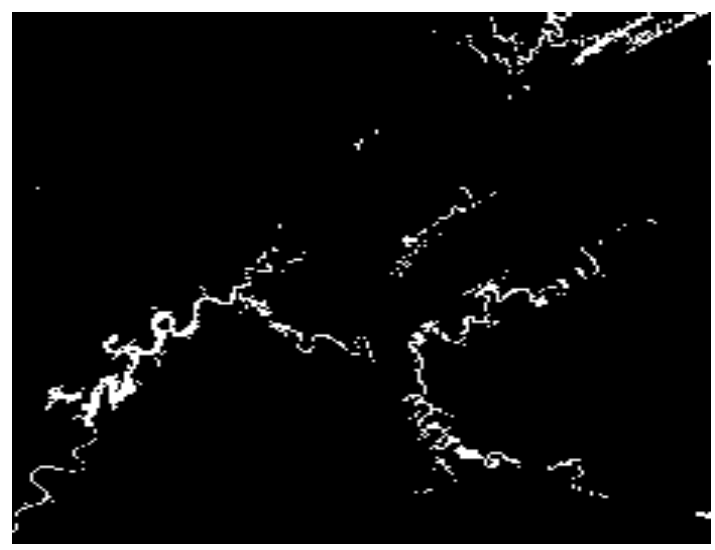

Fig.5 Water Areas Segmented by Color Histogram Method 


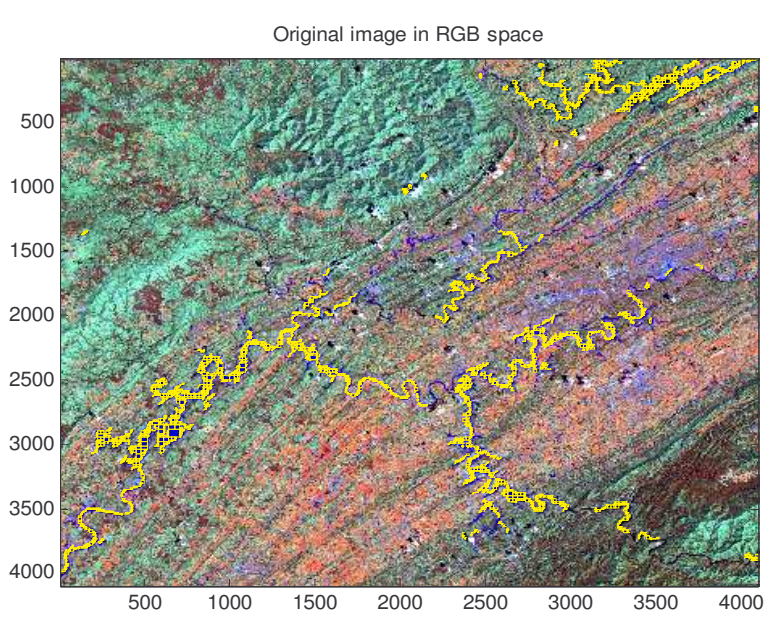

Fig.6 Water Areas (Yellow Squares)

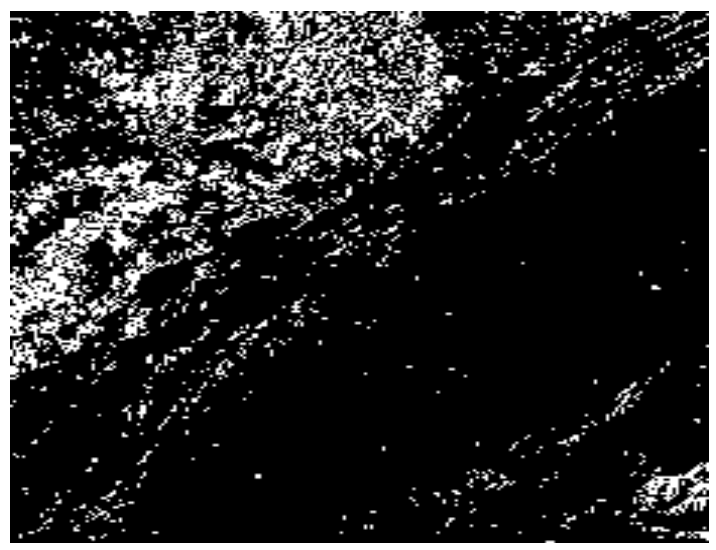

Fig.7 Tree Areas Segmented by Color Histogram Method

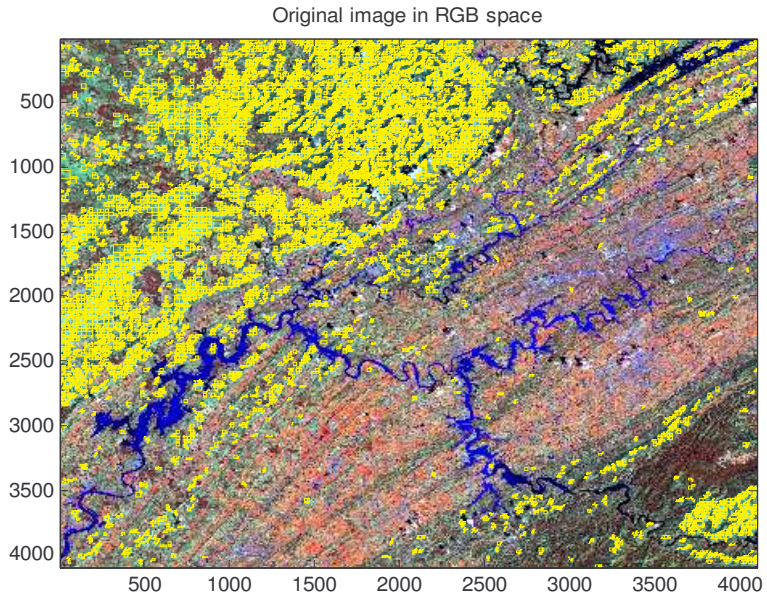

Fig.8 Tree Areas (Yellow Squares)

Considering numerous diversified objects, when $\mathrm{K}$ is a large number, K-Means clustering tends to very time consuming. In order to simplify computational complexity, another example is given in an alternative study.

\section{B. Image Segmentation in a Three-Layer Color Space}

Three-layer case is applied to K-Means clustering using a tristimulus value method which consists of luminosity layer, red-green chromaticity and blue-yellow chromaticity layers. Mahalanobis distance is employed as distance metric which is defined as (6), where $X_{A}$ is cluster center of layer $A, s$ is any pixel, $\mathrm{d}$ is Mahalanobis distance and $\mathrm{K}_{\mathrm{A}}^{-1}$ is inverse of covariance matrix.

$\mathrm{d}=\left(\mathrm{s}-\mathrm{X}_{\mathrm{A}}\right)^{\mathrm{T}} \mathrm{K}_{\mathrm{A}}{ }^{-1}\left(\mathrm{~s}-\mathrm{X}_{\mathrm{A}}\right)$

Winner-take-all method is used for optimal clustering. Its learning network classifies input vectors into one of the three specified number of categories according to clusters detected in the training set. The training is performed in an unsupervised mode. Every cluster center has an associated weight that is referred to as $\mathrm{w}_{\mathrm{i}}$. An input vector $\left[\mathrm{v}_{1}, \mathrm{v}_{2}, \mathrm{v}_{3}\right]$ is selected in the three-dimensional space. The winner has been defined as one whose cluster center is closest to inputs. $\mathrm{w}_{\mathrm{ij}} \mathrm{X}=\min \left(\mathrm{w}_{\mathrm{i}} \mathrm{x}\right)$ for $\mathrm{j}=1,2,3$ and $\mathrm{i}=1,2,3$

Assume cluster center $\mathrm{S}$ wins. Then weight increment of $\mathrm{S}$ is computed as (8), where $\alpha$ is a small positive learning constant. $\alpha$ decreases as competitive learning progresses.

$\Delta \mathrm{w}_{\mathrm{ij}}=\alpha\left(\mathrm{x}_{\mathrm{j}}-\mathrm{w}_{\mathrm{ij}}\right)$, for $\mathrm{j}=1,2,3$ and $\mathrm{i}=1,2,3$

All input data are fed to algorithm repeatedly. Algorithm will identify all three clusters and associate samples with the nearest cluster. This criterion corresponds to finalizing the weight vector that is closest to the input $\mathrm{x}$. Only winner weight vector is adjusted. Given proper initial input vector, after certain loops of iteration, each cluster center converges so as to represent data clusters more accurately.
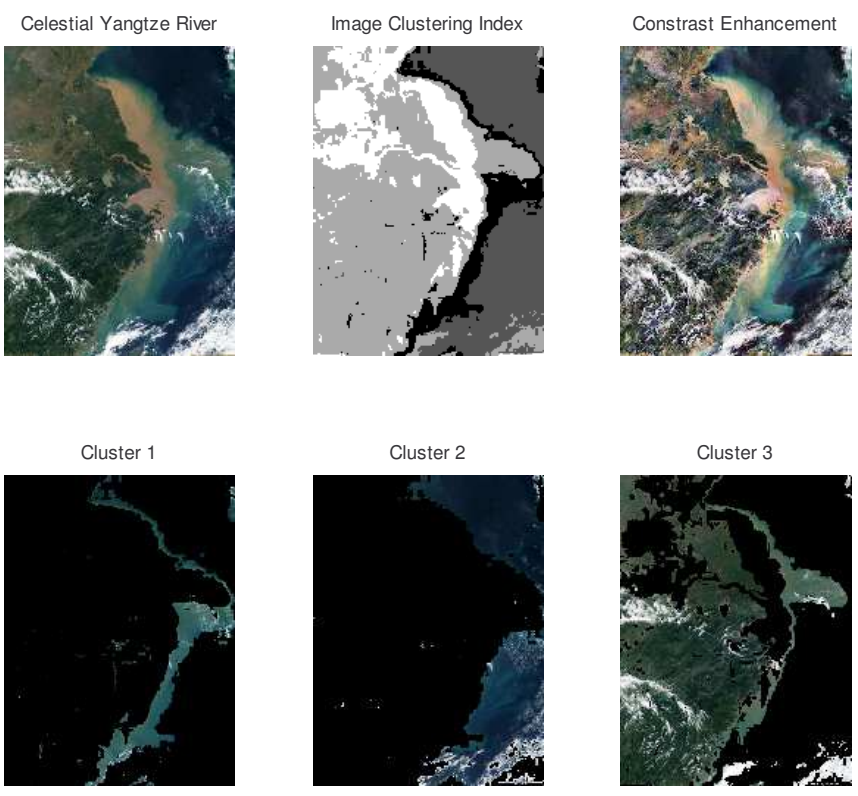

Fig.9 Optimal Clustering of Yangtze River Image 
As a result of clustering procedure, objects within each of three clusters are as close to each other as possible, and as far as possible from objects of other clusters. During the training, dissimilar vectors are rejected thus only one is accepted for weight computing. For each input vector, the $\mathrm{K}-\mathrm{Means}$ clustering returns a clustering index. The winner is the one with the weight vector closest to the input vector.

Contrast stretching is then applied to enhance the critical information from those clusters with important signature right after image segmentation. Decision making depends on the restoration image succeeding a complete clustering procedure. In Fig 9, the original satellite image of Yangtze river, its index for clustering (with 3 clusters), its enhanced image in terms of both K-means clustering and contrast stretching after restoration are shown on the top row. Three separated clusters using tristimulus value method are shown on the bottom row of Fig. 9, where the optimal clustering methodology has been employed.

\section{OPtimal Processing of LASER DOPPLER IMAgING}

There is no doubt that image segmentation using KMeans clustering is capable of analyzing small scale NIR imaging from the laser Doppler scanner system. This type of medical instrumentation is valuable on assessment of burn depth on skin surface. Its principle is as follows. NIR image relates to thermal energy distribution presented by the Doppler blood flow image originated from a laser beam, which leads to a particular pattern. Images are displayed as various colors representing a scale of blood flow. In some areas of medical science, spot measurements have certain limitation against thorough assessment of the whole skin region, so here the pictorial representation and precise numerical measurement of skin blood flow are conducted at the same time. Since it is known that 24-hour is a critical period for the burn event diagnosing and treatment, a rapid and accurate technique for assessing burn depth is necessary. In this case, a fast image processing methodology is extremely substantial. The 2-dimensional NIR image from direct measurement (Fig.10-11) and its generated 3dimensional NIR image (Fig.12-13) have been partitioned into different layers by K-means clustering approaches. All pictures shown result from scanning of normal skin surface. Fig. 11 shows the clustering result of 2D Laser Doppler NIR image of Fig. 10. Fig. 13 shows the clustering result of a generated 3D Laser Doppler NIR image of Fig. 12. Different clusters are discriminated clearly using K-means clustering. For other cases when normal, malignant and abnormal portions of the skin surface occur at the same time, significant mismatch will be identified according to the unusual color variations. Consequently, different types of skin surface regions could be distinguished immediately using this method. In Fig. 11, several color regions are identified on a 2D surface. In Fig.13, several color layers are identified on a $3 \mathrm{D}$ representation of skin surface.

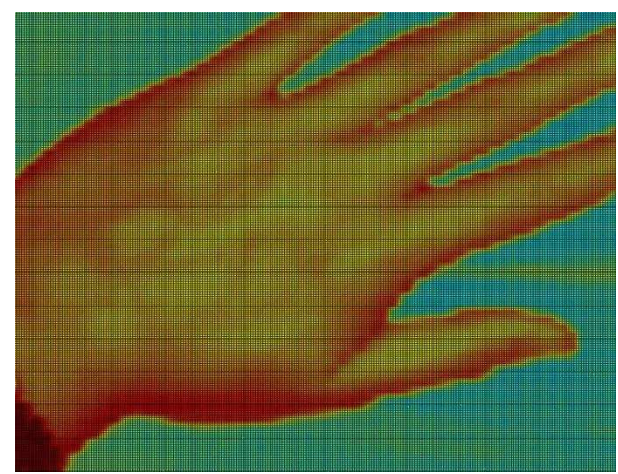

Fig.10 NIR Image from Laser Doppler Scanner (2D)

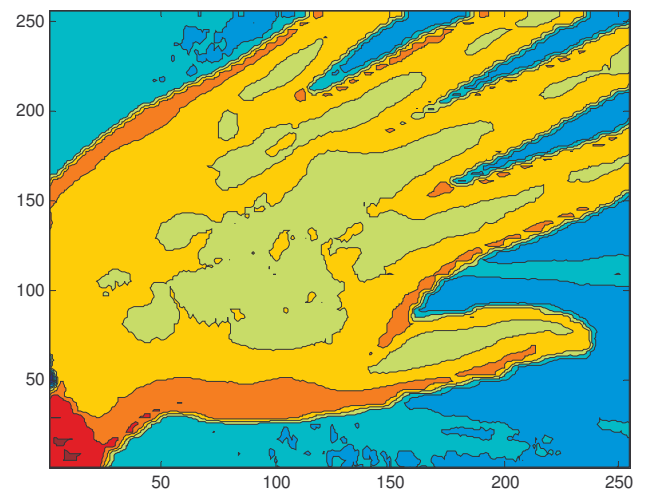

Fig.11 Clustering of NIR Doppler Imaging (2D)

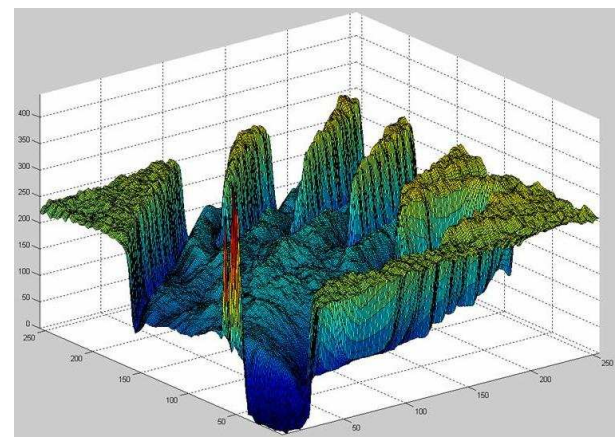

Fig.12 Three-Dimensional NIR Image

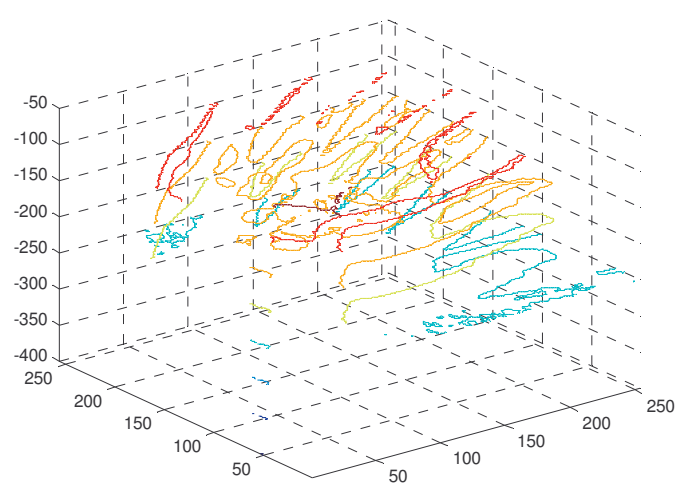

Fig.13 Clustering of NIR Doppler Imaging (3D) 
Now results of medical NIR Doppler imaging for both 2D and 3D cases have been differentiated. In consequence, locations of possible abnormal skin potion can be decided. Besides, via technology integration with other image processing procedures, namely, noise smoothing, contrast stretching, image restoration, etc, an actual image is retrievable from the original NIR based image, where the lowest value corresponds to color of black and the highest value to color of white. The resulting Fig. 14 has shown the hand surface image in an intuitive manner.

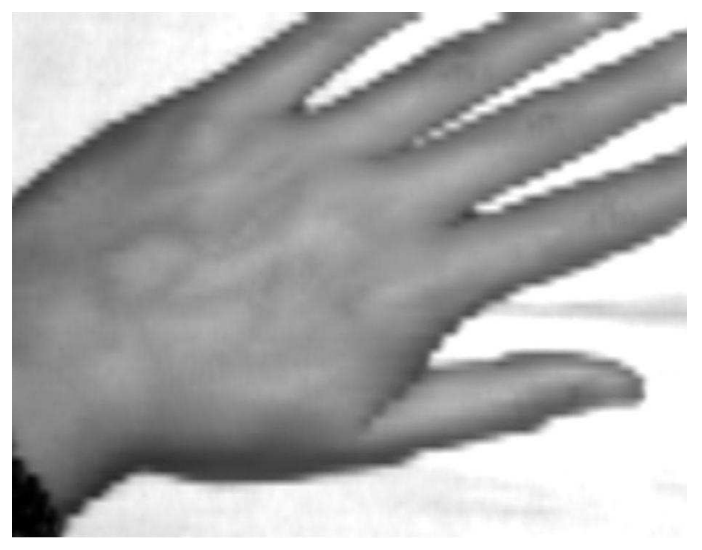

Fig.14 Restoration of NIR Image

\section{CONCLUSION}

This study is concerned with image segmentation in order to extract, analyze and enhance both large scale and small scale IR/NIR images. The global image segmentation via KMeans clustering is proposed for data processing and then some optimal clustering methodologies have been applied. The corresponding desired clusters can be achieved using either object oriented segmentation or spectrum oriented segmentation algorithm. Clustering analysis divides sample data into several groups so that the similar data objects are incorporated together while different data objects are separated apart. Optimal clustering approaches such as nearest neighbor rule and winner-take-all algorithm are conducted along with clustering procedures. Taking into account of the actual complexity of computation, a reduced order K-means clustering is also presented for large scale satellite image processing. The results from various case studies of satellite spatial imaging system and laser Doppler imaging system are shown. This work is a preliminary study for segmentation of IR/NIR images, where thermal sensors are uniquely used for the detection of radiation at the infrared spectrum range. Combined with some other image processing procedures (e.g., contrast stretching), these enhanced images from real scenes can be restored for both large scale and small scale imaging cases by virtue of image retrieval. The real time image processing will be the future research direction.

\section{REFERENCES}

[1] R. Duda, P. Hart, D. Stork, Pattern Classification, $2^{\text {nd }}$ Edition, John Wiley Press, 2001

[2] R. Gonzalez, R. Woods, Digital Image Processing, $2^{\text {nd }}$ Edition, Prentice Hall, 2002

[3] Milan Sonka, Image Processing, Analysis \& Machine Vision, $2^{\text {nd }}$ Edition, Thomson and Cole Press, 1998

[4] Tamel Bose, Digital Signal and Image Processing, John Wiley and Sons Press, 2004

[5] L. Shapiro, G. Stockman, Computer Vision, Prentice Hall Inc, 2001

[6] Simon Hykin, Neural Networks - A Comprehensive Foundation, $2^{\text {nd }}$ Edition, 1999, Prentice Hall

[7] R. Green, M. Shimada, "On-Orbit calibration of a multi-spectral satellite sensor using a high altitude airborne imaging spectrometer", Advances in Space Research, v 19, n 9, 1997, p 1387-98

[8] D. Dantes, C. Renard, et al, "Influence of spectral response dispersion on the performances of infrared imaging systems", Proceedings of SPIE - International Society for Optical Engineering, v3377, 1998, p 97-103

[9] R. Maniewski, A.Liebert, etc, "Selected Applications of Near Infrared Optical Methods in Medical Diagnosis", Opto-Electronics Review.

[10] T. Button, Haifang Li, et al, "Dynamic infrared imaging for the detection of malignancy" Physics in Medicine and Biology, v 49, n 14, 21 July 2004, p 3105-16

[11] R. Samadani, C. Han, "Computer-assisted extraction of boundaries from images" In Proc. SPIE Storage and Retrieval for Image and Video database, 1993

[12] D. Daneels and D. Campenhout, et al. "Interactive outlining: An improve approach using active contours," In Proc. SPIE Storage and Retrieval for Image and Video databases, 1993.

[13] H. Osman, S. Blostein, "SAR image processing using probabilistic winner-take-all learning and artificial neural networks", Proceedings of International CONF on Image Processing, p 613-616, vol.2, 1996 